\title{
FATORES DE RISCO PARA O ESÔFAGO DE BARRET: um estudo retrospectivo
}

Carolina Oliveira BERNARDES ${ }^{1}$

Laura Inês de Oliveira SANTOS ${ }^{2}$

Aline Chagas PRADO 3

Maria Cristina TERÇONI ${ }^{4}$

Caroline Nogueira TERRA ${ }^{5}$

Juliana TAVARES ${ }^{6}$

Vanessa Cristina FARIA ${ }^{7}$

Isabela de OLIVEIRA ${ }^{8}$

Luiz Carlos de ANDRADE ${ }^{9}$

Roberta Bessa Veloso SILVA ${ }^{10}$

Eric Batista FERREIRA ${ }^{11}$

1,2,3,4,5,6,7,8 Acadêmicos do curso de Medicina, Universidade José do Rosário Vellano/UNIFENAS

${ }^{9}$ Professor e médico gastroenterologista do Hospital Universitário Alzira Velano/HUAV/UNIFENAS e-mail: endoscopia.huav@unifenas.br

${ }^{10}$ Doutora em Estatística e Experimentação Agropecuária/UFLA, Universidade José do Rosário Vellano/UNIFENAS e-mail: bessaveloso@yahoo.com.br

${ }^{11}$ Doutor em Estatística e Experimentação Agropecuária/UFLA, Universidade Federal de Alfenas/UNIFAL e-mail: eric.ferreira@unifal-mg.edu.br

Recebido em: 08/07/2016 - Aprovado em: 10/09/2016 - Disponibilizado em: 18/12/2016

\section{RESUMO}

O esôfago de Barrett (EB) resulta de complicação da Doença do Refluxo Gastroesofágico (DRGE) de longa duração. A grande preocupação quanto ao esôfago de Barrett é o risco de progressão para o câncer, principalmente o adenocarcinoma, que é considerado um tumor letal, com uma taxa de sobrevivência, em cinco anos de $20 \%$. Sendo assim, esta displasia constitui-se o principal marcador biológico preditivo de evolução para adenocarcinoma. Identificar e graduar a displasia são importantes questões na prática diagnóstica. Os fatores de risco para desenvolvimento do EB são os que favorecem o refluxo gastroesofágico. Diante disso, este trabalho teve como objetivo determinar a prevalência de esôfago de Barret e sua relação com refluxo gastresofágico, bem como com o adenocarcinoma esofágico, no Hospital Universitário Alzira Vellano - HUAV, no município de Alfenas, MG. Após a coleta das informações, os dados foram organizados para a realização da análise estatística. Foi utilizado o teste exato de Fisher, ao nível nominal de 5\% de significância. Pode-se concluir que não foi evidenciado uma associação significativa entre o esôfago de Barret e a DRGE. Também não foi observada uma relação entre o EB e o adenocarcinoma esofágico. Porém, verificou-se uma associação significativa entre o adenocarcinoma esofágico e o gênero; e a DRGE e o gênero.

Palavras-chave: DRGE, Adenocarcinoma esofágico, Esôfago, Metaplasiaintestinal.

\section{ABSTRACT}

Barrett's esophagus (BE) results from complication of gastroesophageal reflux disease (GERD) long term. The major concern as to Barrett's esophagus is the risk of progression to cancer, especially adenocarcinoma, which is considered a lethal tumor, with a survival rate at five years of $20 \%$. Thus, this dysplasia constitutes the main biological marker of 
progression to adenocarcinoma. Identify and grade dysplasia are important issues in the diagnostic practice. Risk factors for development EB are favoring gastroesophageal reflux. Whereupon, this study aimed to determine the prevalence of Barrett's esophagus and its relation with gastroesophageal reflux, as well as esophageal adenocarcinoma, the University Hospital AlziraVellano - HUAV in the city of Alfenas, MG. After collecting the information, the data were organized for statistical analysis. Fisher's exact test was used, the nominal 5\% level of significance. It can be concluded that it was not shown a significant association among Barrett's esophagus and GERD.No relation among the EB and esophageal adenocarcinoma was observed. However, verified a significant association among esophageal adenocarcinoma and gender; and GERD and gender.

Keywords:GERD, Esophagealadenocarcinoma, Esophagus, Intestinal metaplasia.

\section{Introdução}

O esôfago de Barrett (EB) resulta de complicação da Doença do Refluxo Gastroesofágico (DRGE) de longa duração. Caracteriza-se pela presença de mucosa tipogástrica, glandular, em órgão tubular, cujas biópsias confirmem a presença de metaplasia intestinal.

O deslocamento proximal da junção escamocolunar em relação à junção esofagogástrica é vistohistologicamente, pela substituição do epitélio estratificado pavimentoso por epitélio colunar especializado com células caliciformes,(CAPPELLANES, 2009).

A metaplasia intestinal é um processo de transformação de um tipo de tecido totalmente adulto e diferenciado, para outro tipo de tecido igualmente adulto e diferenciado de mesma linhagem, e que pode evoluir para uma displasia. Esta última, tratase de uma organização anormal ou diferenciação desordenada de células ou tecido presente em um órgão, envolve alterações fenotípicas e genotípicas, sendo considerada uma lesão pré-maligna, (CAPPELLANES, 2009).
A grande preocupação quanto ao esôfago de Barrett é o risco de progressão para o câncer, principalmente o adenocarcinoma, que é considerado um tumor letal, com uma taxa de sobrevivência em cinco anos de 20\%. Sendo assim, esta displasia constitui-se o principal marcador biológico preditivo de evolução para adenocarcinoma. Identificar e graduar a displasia são importantes questões na prática diagnóstica (MUTHUSAMY, et al., 2015).

O diagnóstico de esôfago de Barrett (EB) deve ser feito através de exame endoscópico e anatomopatológico. A endoscopia com biópsia da zona aparentando ser epitélio gástrico no esôfago distal e, para confirmação de $\mathrm{EB}$, é necessária a presença de metaplasia intestinal à biópsia. Sabe-se, no entanto, que não é sempre que esses métodos diagnósticos conseguem confirmar o EB, com evolução displásica(CAPPELLANES, 2009).

Segundo Muthusamyet al. (2015), oesôfago de Barrett possui um potencial de degeneração maligna que varia de 2 a 40\%, de acordo com vários fatores como extensão do tecido metaplásico, grau de displasia, duração da doença e alterações genômicas. 
Está mais associado ao sexo masculino, entre 50 a 70 anos, com história prévia de DRGE há mais de 10 anos. Estimase que o risco de desenvolver adenocarcinoma seja de 30 a 125 vezes maior ao se comparar como da população em geral.

Dessa forma, faz-se necessário analisar e discutir as associações epidemiológicas com esôfago de Barrett, dado o seu alto risco de evoluir com neoplasia (MUTHUSAMY,et al., 2015).

A Doença do Refluxo Esofágico(DRGE) é caracterizada pela substituição do epitélio estratificado pavimentoso do esôfago por epitélio colunar contendo células intestinalizadas em resposta à agressão pelo ácido (metaplasia intestinal) (HAGGITT, 1994).

De acordo com Jones (2002), é importante salientar que o esôfago distal revestido por epitélio colunar sem metaplasia intestinal não é classificado esôfago de Barret, porém $20 \%$ desses casos podem ter o diagnóstico de esôfago de Barret em exames subsequentes.

Além disso, há situações em que o aspecto endoscópico da transição do esôfago para o estômago mostra-se normal, mas há metaplasia revelada pela biopsia. Isso também não deve ser interpretado como esôfago de Barret.

Essa condição patológica pode ser classificada de acordo com sua extensão em: esôfago de Barret curto (metaplasia inferior a $3 \mathrm{~cm})$ e "Barret longo ou clássico" se a extensão for maior ou igual a $3 \mathrm{~cm}$. Aproximadamente, 3 a $5 \%$ dos pacientes portadores de DRGE possuem esôfago de Barret curto e 10 a 15\% apresentam "Barret longo ou clássico", (MANN, 1989).

No Brasil, há poucos estudos epidemiológicos sobre esôfago de Barret. Acredita-se que a incidência real dessa condição na população seja subestimada pela existência de indivíduos assintomáticos ou oligossintomaticos; avaliação parcial pelo exame endoscópico e casos de EB detectados pelos sintomas de adenocarcinoma, (ANDREOLLO,1997).

É sabido que a agressão do esôfago ocasionada pelo ácido ou refluxo duodenogastrico leva a célula a se diferenciar no fenótico de Barret. Entretanto, esse mecanismo não é totalmente esclarecido e há diferentes teorias para explicar a origem das células-tronco pluripotentes.

A mais aceita é a "teoria da metaplasia do novo", onde surgiria da papila inflamada do epitélio escamoso. Há também a "teoria da célula ductalmetaplasica" (células-tronco originariam das glândulas submucosas esofágicas) e a teoria que sugere que células da junção do esôfago e estomago colonizariam o esôfago distal (NIEMANTSVERDRIET, 1997). 
Os fatores de risco para desenvolvimento do EB são os que favorecem o refluxo gastroesofágico: incompetência do esfíncter inferior do esôfago, amplitude de contração no esôfago distal menor, hérnia de hiato e o refluxo do conteúdo duodenogástrico(NIEMANTSVERDRIET, 1997).

Segundo Reynolds (2002), aproximadamente, de 6 a $20 \%$ dos pacientes são assintomáticos. Os que apresentam sintomas, em geral, são os mesmos com DRGE não complicada, com queixas dispépticas e manifestações otorrinolaringológicas ou pulmonares.

Sintomas com duração superior a5 anos, regurgitação noturna e pirose são fatores de risco. A incidência é maior entre a faixa etária de 60 a 70 anos, mais predominante no sexo masculino, caucasianos, hispânicos, classe social baixa e portadores de obesidade.

Cerca de $10 \%$ dos pacientes portadores de EB desenvolvem adenocarcinoma no terço inferior do esôfago. Conforme mencionado acima, não se conhece o número real de pacientes com EB, portanto, as estimativas para o risco de câncer são imprecisas, (REYNOLDS, 2002).

De acordo com Moraes Filho,et al. (2002), a endoscopia é fundamental no diagnóstico do esôfago de Barret. A observação do epitélio semelhante a mucosa gástrica recobrindo, parcialmente ou totalmente, a porção proximal à junção esofagogástrica é chave no diagnóstico.

O diagnóstico é a presença de mucosa colunar entre a extremidade proximal das pregas gástricas e o início da junção escamocelular mediante confirmação histopatológica.

$$
\text { Segundo Lambert (2002) para }
$$
conseguir uma maior acurácia no diagnostico endoscópico vários corantes têm sido utilizados, associados ou não com novas tecnologias (como a endoscopia com magnificação de imagens).

Como exemplo dessas substancias há o índigo de carmim, que auxilia na análise da arquitetura do epitélio colunar e dirigir a biopsia na suspeita de neoplasias localizadas em áreas com metaplasia intestinal. Lugol a $2 \%$, que localiza e identifica irregularidades na linha de junção escamocolunar nos segmentos curtos do esôfago de Barret; a violeta cristal ou a violeta de cresyl que também realça o epitélio colunar e o azul de metileno que tinge de azul as células caliciformes (LAMBERT, 2002).

Em relação ao azul de metileno, muito utilizado hoje em dia, a coloração pode sofrer modificações conforme a intensidade da displasia. Quando há segmentos longos de esôfago de Barret, sem displasia, as células colunares e caliciformes do epitélio intestinal absorvem muito o corante e se tornam azul escuro de forma homogênea. Em 
adenocarcinomas ou displasias de grande intensidade há menos células caliciformes e a coloração é azul claro (CANTO, 2001).

Entretanto, estudos feitos por Dave,et al. (2001) mostraram baixa sensibilidade e especificidade na utilização desse corante para avaliar áreas de displasias ou adenocarcinomas.

As biopsias para a realização do exame histológico devem ser realizadas em áreas com suspeita de presença de epitélio colunar e na extremidade proximal da projeção digitiforme.

Quando há presença de metaplasia intestinal é confirmado o diagnóstico de esôfago de Barret A avaliação histopatológica é o preditor mais importante para análise do risco do EB evoluir para adenocarcinoma,

O objetivo preconizado é o mesmo que o da DRGE e visa controlar os sintomas (se houver) e manter a mucosa cicatrizada. Do ponto de vista prático, o tratamento tem como foco a redução do potencial agressivo do conteúdo gástrico. Para isso, o controle do refluxo é fundamental, (MORAES FILHO, et al., 2002).

Conforme Moraes Filho, et al. (2002), o tratamento não farmacológico deve ser implementado simultaneamente ao farmacológico. Algumas medidas são apresentadas a seguir:

- Elevação da cabeceira da cama $(15 \mathrm{~cm})$;
- Moderar a ingestão dos seguintes alimentos, na dependência da correlação com os sintomas: gordurosos, cítricos, café, bebidas alcóolicas, bebidas gasosas, menta, hortelã, produtos de tomate, chocolate;

- Cuidados especiais para medicamentos potencialmente de risco, como anticolinérgicos, teofilina, antidepressivos tricíclicos, bloqueadores de canais de cálcio, agonistas beta adrenégicos, alendronato;

- Evitar deitar-se nas 2 horas após a refeição;

- Evitar refeições copiosas;

- Redução drástica ou cessação do fumo;

- Reduzir o peso corporal (emagrecimento).

No tratamento farmacológico os medicamentos mais utilizados são os inibidores da bomba de prótons (IBP), que aliviam os sintomas do refluxo e cicatrizam a esofagite mais rapidamente que os antagonistas $\mathrm{H}_{2}\left(\mathrm{AH}_{2}\right)$.

Entretanto, a dose nem sempre é suficiente para normalizar o refluxo em todos os pacientes (há o escape do ácido noturno). Dessa forma, propõem-se a administração de dose dobrada (o dobro da dose plena para as esofagites mais graves (CHIBA, 1997).

De acordo com Langman (2000), Inibidores da COX-2 também possuem resultados satisfatórios na redução do risco de câncer de esôfago. O mecanismo da proteção é a diminuição da expressão da COX-2 que 
pode aumentar a progressão de neoplasia no EB. Além disso, há diminuição da 15lipooxigenase-1 e a liberação de prostraglandina E2.

Outra modalidade de tratamento é o endoscópico, que pode ser realizado por mucosectomia e ablação. A mucosectomia é uma terapêutica que permite a remoção de lesões gastrointestinais presentes nas camadas superficiais da parede do tubo digestivo (camada mucosa e parte da submucosa).

O procedimento é curativo quando a lesão é superficial, as margens estão livres de tumor e a neoplasia é bem diferenciada. (NIGRO, 1999).

O tratamento cirúrgico por fundoplicatura é capaz de prevenir o refluxo ácido e duodenoesofagogastrico. Entretanto, foi demonstrado que tanto o tratamento com IBP em altas doses quanto afundoplicatura conseguiu diminuir a metaplasia. Como prova, foram relatados na literatura vários casos de adenocarcinoma após fundoscopia (CSENDS, 2002).

Além disso, o diagnóstico do adenocarcinoma esofágico em fases iniciais permite intervir antecipadamente à invasão local e à ocorrência de metástases, aumentando as chances de o tratamento ser curativo. (QUEIROGA; PERNAMBUCO, 2006).

Diante disso, este trabalho teve como objetivos determinar a prevalência de esôfago de Barret e sua relação com refluxo gastresofágico, bem como a evolução para adenocarcinoma, no Hospital Universitário Alzira Vellano - HUAV, no município de Alfenas, MG.

\section{Material e Métodos}

Foi desenvolvido um estudo transversal com base no levantamento de biopsias realizadas no Hospital Universitário Alzira Velano - HUAV, durante o período de 2009 a 2014. Portanto, foram analisados 656 laudos disponíveis no SAME deste hospital, no município de Alfenas-MG.

Após a coleta das informações, os dados foram organizados para a realização da análise estatística. Foi utilizado o teste exato de Fisher, ao nível nominal de $5 \%$ de significância (FERREIRA, 2011).

As principais hipóteses testadas estão apresentadas a seguir.

$\mathrm{Na}$ primeira etapa, foi feita a associação entre o esôfago de Barret com as variáveis de interesse (gênero, DRGE, adenocarcinoma esofágico). Além disso, tevese o interesse em verificar uma possível relação entre DRGE vs gênero; adenocarcinoma esofágico vs gênero, adenocarcinoma esofágico vs DRGE.

Para análise dos dados foi utilizado o teste exato de Fisher, ao nível nominal de 5\% 
de significância. As análises foram realizadas no software R (R CORE TEAM, 2016).

\section{Resultados e Discussão}

Na Tabela 1 está apresentada os resultados do teste exato de Fisher, ao nível nominal de 5\% de significância. Verifica-se que houve associação significativa entre DRGE e gênero $(p=0,0346)$.

Tabela 1. Teste exato de Fisher, ao nível nominal de $5 \%$ de significância de acordo com as relações entre o Esôfago de Barret (EB) e as variáveis de interesse

\begin{tabular}{c|c}
\hline Variáveis de interesse & $P$-valor \\
\hline EB $v s$ gênero & $0,5686 n s$ \\
\hline EB $v s$ DRGE & $0,6709 n s$ \\
\hline EB vsadenocarcinoma esofágico & $1,0000 n s$ \\
\hline DRGE $v s$ gênero & $0,0346^{*}$ \\
\hline Adenocarcinoma esofágico $v s$ & $0,0145^{*}$ \\
gênero & \\
\hline Adenocarcinoma esofágico $v s$ & $0,5525 n s$ \\
DRGE & \\
\hline
\end{tabular}

*Significativo ao nível nominal de 5\% de significância, $(\mathrm{p}<0,05)$

${ }^{n s}$ Não significativo ao nível nominal de $5 \%$ de significância, $(p>0,05)$

Observou-se que a prevalência de DRGE no gênero feminino é 1,5 vezes do que no gênero masculino, (resultados não apresentados).

A relação entre adenocarcinoma esofágico e o gênero mostrou-se significativa, ( $\mathrm{p}=0,0145)$, conforme está apresentado na Tabela 1.

A prevalência deste câncer no gênero masculino foi aproximadamente 2 vezes maior se comparado ao gênero feminino, (resultados não apresentados).

Muthusamyet al. (2015), observou que oesôfago de Barrettestá mais associado ao sexo masculino, entre 50 a 70 anos, com história prévia de DRGE há mais de 10 anos.

De acordo com a Tabela 1, verifica-se que não houve associação significativa entre o esôfago de Barret e o gênero, $(\mathrm{p}=0,5686)$.

Quanto às possíveis associações entre o esôfago de Barret e as variáveis de interesse, pode-se observar, na Tabela 1 , que estas relações não foram significativas, $(\mathrm{p}>0,05)$.

Não foi evidenciado, neste trabalho, que o esôfago de Barret e adenocarcinoma esofágico estão relacionados, $(p=1,000)$, conforme mostra a Tabela 1 .

Pode-se observar, na Tabela 1 , não houve associação significativa entre o esôfago de Barret e a DRGE, na amostra de registros selecionados, $(\mathrm{p}=0,6709)$.

Ao comparar estes resultados com àquelesobtidos por Jones (2002), verificou-se que estes foram contraditórios, uma vez que o esôfago de Barrett (EB) resulta de complicação da Doença do Refluxo Gastroesofágico (DRGE) de longa duração, conforme Jones (2002). 


\section{Conclusão}

Pode-se concluir que não foi evidenciado uma associação significativa entre o esôfago de Barret e a DRGE. Também não foi observada uma relação entre o EB e o adenocarcinoma esofágico, bem como o EB e o gênero.

Quando o gênero foi associado aoadenocarcinoma esofágico verificou-se uma relação entre eles; além de uma associação significativa entre a DRGE e o gênero.

\section{Referências Bibliográficas}

ANDREOLLO, N. A. et al. Incidência e epidemiologia do epitélio de Barrett no Gastrocentro/Unicamp.ArqGastroenterol, v. 34, p. 22-6, 1997.

CANTO, M. I.; SETRAKIAN, S.; WILLIS, J. E; CHAK, A.; PETRAS, R. E.; SIVAK, M. $\mathrm{V}$. Methilene blue staining of dysplastic and nondysplastic Barrett's esophagus: an in vivo and ex vivo study. Endoscopy, 33:391-400, 2001.

CAMERON, A. J.; ZINMEISTER, A. R; BALLARD, D. J; et al. Prevalence of columnar-lined (Barrett's) esophagus: comparison of population based clinical and autopsy findgins. Gastroenterology, 99:9118-9122, 1990.

\section{CAPPELLANES, C. A. Projeto Diretrizes} Sociedade Brasileira de Endoscopia Digestiva. Gestão2009.

CHIBA, N.; GARA, C. J. de; WILKINSON, J. M.; HUNT, R. H. Speed of healing and symptom relief in grade II to IV gastroesophageal reflux disease: a meta- analysis. Gastroenterology, 112:1798-810, 1997.

CSENDS, A.; BURDILES, P.; BRAGHETTO, I., et al. Dysplasia and adenocarcinoma after Barrett's esophagus: the need for long-term subjective and objective follow-up. Ann Surg, 235:178-185, 2002.

DAVE, U.; SHOUISA, S.; WESTAY, D. Methilene blue staining: is it really useful in Barret's esophagus? Gastrointest Endosc,;53:333-5, 2001.

FERREIRA,D.F. Estatística

Básica.Lavras:Editora UFLA,2009, 2a ed. rev. $664 \mathrm{p}$.

HAGGITT, R. C. Barrett's esophagus, dysplasia and adenocarcinoma.Hum Pathol, v. 25, p. 982-93, 1994.

JONES, T.; SHARMA, P.; WESTON, A., et al. Yield pf intestinal metaplasia in patients with suspect short segment Barrett's esophagus (SSBE) on repeat endoscopy.Dig Dis Sci, 47:2108-2111, 2002.

LAMBERT, R. Diagnosis of esophagogastric tumors. Endoscopy, 34:129-38, 2002.

LANGMAN, M.; CHENG, K.; GILMAN, E. Lancashire R. Effect of anti-inflammatory drugs on overall risk of commom cancer: case-control study in general practice research database. $\mathrm{BMJ} ; 320: 1,642-646$, 2000.

MANN, N. S; TSAI, M. F; NAIR, P. K. Barrett's esophagus in patients with symptomatic reflux esophagitis.Am J Gastroenterol, 84:1494-1496, 1989.

MORAES FILHO, J. P. P.; CECCONELLO, I.; GAMA, R. J.; CASTRO L. P.; HENRY, M.A. Consensus Group. Brazilian consensus on gastroesophageal reflux 
disease: proposals management. Am J gastroenterol, 97:241-248, 2002.

MUTHUSAMY, V. R.; KIM, S.;

WALLACE, M. B. Advanced imaging in

Barret's Esophagus.GastroenterolClin North

Am, Jun, 44(2):439-58, 2015.

NIEMANTSVERDRIET, E.; TIMMER, R.;

BREUMELHOF, R.; SMOUTH, A. J. P.

$M$.The roles of excessive gastroesophageal reflux, disordered o esophageal motility and decrease mucosal sensitivity in the pathogenesis os Barrett's oesophagus.Eur J GastroenterolHepatol, ;9:515-519, 1997.

NIGRO, J. J.; HAGEN, J. A.; DEMEESTER, $\mathrm{T}$.; et al. Occult esophageal adenocarcinoma: extent of disease and implications for effective therapy.Ann Surg; 230:433-440, 1999.

QUEIROGA, R. C.; PERNAMBUCO, A. P. Câncer de esôfago: epidemiologia, diagnóstico e tratamento. Revista Brasileira de Cancerologia, p. 173-178, 2006.

REYNOLDS, J. C; RAAHIMI, P.; HIRSCHL, D. Barrett'sesophagus: clinicalcharacteristics.GastroenterolClin N Am; 31:441-460, 2002.

R CORE TEAM. R: A language and environment for statistical computing. $R$ Foundation for Statistical Computing, Vienna, Austria. 2016. 